\title{
Applications of Statistical Methods
}

GECTIONS $A^{*}$ (Mathematics) and $J$ (Psychology) $S$ of the British Association held a joint discussion at Leicester on September 12 on "The Validity and Value of Methods of Correlation", which was opened by Prof. C. Spearman. One group of speakers dealt with statistical methods from a general point of view, with applications to a wide field, including medical and sociological aspects, while a second group restricted themselves to psychological applications, and in particular to Spearman's 'two-factor theory'. It will be simpler to separate these two groups here, although in the actual discussion they were mingled in a rather confusing manner.

Dr. S. Dawson (Glasgow) gave a warning against the misuse of statistical measures such as correlation coefficients, which, as he showed by an example, can be sometimes very misleading. The statistician must not be content with such short cuts, but must be prepared to look beyond his coefficient to the actual data themselves. Dr. Dawson illustrated how this could be done by a reference to an investigation into Glasgow housing conditions.

Dr. S. S. Wilks (Princeton) explained a new criterion for testing the mutual independence of several sets of traits. This criterion may be regarded as a generalisation of $1-R^{2}$, where $R$ is the multiple correlation coefficient between one variate and several others. A full account of the subject will be found in a recent number of Biometrika.

Dr. J. O. Irwin (London) pointed out that there is a tendency for everyone to use coefficients of association, whether he understands them or not. It is desirable not to rely solely upon such a coefficient, but to express results in a form which gives a physical or concrete meaning; the more easily the result may be understood by an intelligent layman the better. Dr. Irwin then applied his principles to several examples, including an investigation into accident proneness at a naval shipyard, carried out for the Industrial Health Research Board. The results were illustrated graphically.

Prof. R. A. Fisher (Rothamsted and London) summed up the discussion of this group of speakers. $\mathrm{He}$ emphasised that no data can absolutely prove a hypothesis, but they can disprove it, and it is the function of the statistician to devise tests which shall indicate clearly when a hypothesis is to bo rejected. For this purpose the statistician must know something about the quality of the experiments, and it is advisable for the experimenter to consult him before fixing the exact design of his experiments.

Before summarising the remarks of the second group of speakers, who dealt with Spearman's 'twofactor theory', it may be well to outline the main points of this theory. No such outline was given at the discussion ; in recent years the theory has become very widely known, and hence the speakers considered themselves justified in dealing only with its more obscure parts.

It is an experimental fact that if a number of dissimilar mental tests are applied to a group of individuals, and the coefficients of correlation between these tests are measured, then these coefficients, taken in sets of four, furnish what are called tetrad differences, the numerical values of which are approximately zero. From this is inferred, by somewhat difficult reasoning, that the marks obtained by an individual in any particular test are the sum of two parts, one proportional to his general factor $g$, and the other proportional to his specific factor $s$ for that particular test. It is tempting, but venturesome, to identify $g$ with general intelligence or general mental energy, while $s$ may be identified with special ability for some particular task.

Prof. C. Spearman (London) mentioned that he had recently returned from a tour of the United States, where he attended a number of psychological meetings in various centres, collecting all the objections raised to the two-factor theory. $\mathrm{He}_{\Theta}$ had tabulated these and indicated the appropriate reply in each case (a copy of this document was given to all present). He then dealt in detail with an objection raised by Prof. E. B. Wilson, that the value of $g$ would be altered by what mathematicians call a linear transformation of the test scores. The reply is that this can only be done by introducing into each of the composite abilities negative elements exactly equal in influence to the positive ones, which is in flagrant contradiction to psychological experience.

A short but vigorous discussion followed. Dr. J. Wishart (Cambridge) pointed out that linear trans. formations of Wilson's type are never used by psychologists, so they need not worry over the difficulties that would be caused if they did use them.

Dr. W. Brown (Oxford and London) presented the results of an extensive research by Dr. W. Stephenson and himself, to test, with every precaution called for by the critics of previous investigations, the abilities of a large number of boys, all of nearly the same age, by means of nineteen carefully selected mental tests. The resulting tetrads were fitted to a smooth curve (exhibited on a large diagram at the meeting) and shown to conform closely with the distribution which is to be expected from random sampling if Spearman's theory is true.

Dr. Wishart dealt searchingly with the question of random sampling errors. He pointed out the great difficulties in deciding whether a distribution of tetrads, such as Dr. Brown's, does really agree with that to be expected from random sampling. Possibly some of the difficulties can be overcome by using a tetrad of product moments in place of that of corre. lation coefficients. Further work on these lines is in progress.

Prof. H. T. H. Piaggio (Nottingham) analysed the results of Dr. Brown and others by the aid of a formula giving $g$ in terms of the test results, but also containing an indeterminate portion. To make this portion negligible we need tests with a greater $g$-saturation. The same formula can be used to discuss the transformations dealt with by Prof. Spearman.

The discussion concluded with forty minutes of rapid questions and answers, which cleared up many points, and perhaps was the most valuable part of the proceedings. The collaboration of workers in two branches of science is not yet so common as it should be, but it is gratifying to notice that it appears to be on the increase.
H. T. H. Praggio. 